\title{
An improved age invariant face recognition using data augmentation
}

\author{
Kennedy Okokpujie ${ }^{1}$, Samuel John², Charles Ndujiuba ${ }^{3}$, Joke A. Badejo ${ }^{4}$, Etinosa Noma-Osaghae ${ }^{5}$ \\ 1,4,5 Department of Electrical and Information Engineering, Covenant University, Ota, Nigeria \\ ${ }^{2}$ Department of Electrical and Electronic Engineering, Nigerian Defence Academy, Kaduna, Nigeria \\ ${ }^{3}$ Department of Electrical and Electronic Engineering, Air Force Institute of Technology, Kaduna, Nigeria
}

\begin{tabular}{l} 
Article Info \\
\hline Article history: \\
Received Jan 28, 2020 \\
Revised Mar 23, 2020 \\
Accepted Jul 13, 2020 \\
\hline Keywords: \\
Age invariant face recognition \\
Data augmentation \\
FG-net aging dataset \\
Inception-ResNet-v2 \\
Noise image augmentation
\end{tabular}

Corresponding Author:

\section{Kennedy Okokpujie,}

Department of Electrical and Information Engineering,

Covenant University,

Km 10 Idiroko Road, Ota, Ogun State, Nigeria.

Email: kennedy.okokpujie@covenantuniversity.edu.ng

\begin{abstract}
In spite of the significant advancement in face recognition expertise, accurately recognizing the face of the same individual across different ages still remains an open research question. Face aging causes intra-subject variations (such as geometric changes during childhood \& adolescence, wrinkles and saggy skin in old age) which negatively affects the accuracy of face recognition systems. Over the years, researchers have devised different techniques to improve the accuracy of age invariant face recognition (AIFR) systems. In this paper, the face and gesture recognition network (FG-NET) aging dataset was adopted to enable the benchmarking of experimental results. The FG-Net dataset was augmented by adding four different types of noises at the preprocessing phase in order to improve the trait aging face features extraction and the training model used at the classification stages, thus addressing the problem of few available training aging for face recognition dataset. The developed model was an adaptation of a pre-trained convolution neural network architecture (Inception-ResNet-v2) which is a very robust noise. The proposed model on testing achieved a $99.94 \%$ recognition accuracy, a mean square error of 0.0158 and a mean absolute error of 0.0637 . The results obtained are significant improvements in comparison with related works.
\end{abstract}

This is an open access article under the CC BY-SA license.

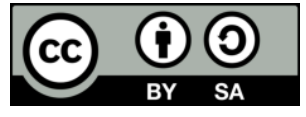

\section{INTRODUCTION}

The need for automated human face recognition cannot be overemphasized as it is required for identification and authentication in various real-life applications. Examples of these applications include border control, voting systems, health care, attendance capturing, and access control. The variant nature of the face with the passage of time has been found from rigorous research to be responsible for the intra-class variations that make facial recognition systems to return a non-match for genuine users. This factor is called "aging" and it makes matching of "query face templates" with stored templates of users' faces in databases unreliable and insecure.

It is generally accepted that the use of deep learning for face recognition application was possible due to many factors of which data augmentation [1] is a part. Face recognition is affected negatively by synthetic makeup and research has shown that synthetic makeup is one of the reasons why celebrities have trouble with face recognition systems. The lifestyle of celebrities usually involves a lot of activities that require several unique synthetic makeups. This causes serious ambiguity issues in face recognition. 
The artificial colors, contouring and uneven skin tone associated with artificial makeovers pose a challenging problem in face recognition that some researchers [2] have attempted to solve using deep convolutional neural networks with promising results. The application of convolutional neural network (CNN) to solve the facial artificial makeup challenge involved the use of augmented pictures of subjects with various types of artificial makeovers.

In some instances, the input images were split into two categories, a category with closely matched pairs of face images and the other categories were unmatched pairs. The goal was to increase the disparity in the unmatched face image pairs and the similarity in the matched face image pairs [3]. The face images of the subjects were fed into CNN networks in pairs with the aim of reducing false matches and increasing true matches across age groups. The age-invariant face recognition was achieved using distance metrics on well-ordered pairs of matched and unmatched face images. Standard databases such as the Face and Gesture Recognition Network Aging Database (FG-NET AD) [4], the MORPH database, the CAD database, the Asian Face Age Dataset (AFAD) and a lot more have been the pool from which researchers working on age-invariant face recognition systems get face images to augment. These standard aging databases provide a platform to standardize research outputs on age-invariant face recognition. The majority of the databases have a limited number of face images that are not large enough for deep learning applications and are thus augmented using a large array of standardized data augmentation processes.

Some researchers use unique face images from subjects who volunteer to have their face image used for age-invariant face recognition research [5]. The aim is usually to get face images from subjects that cover a minimum of twenty to thirty percent of a subject's lifetime. These images are augmented and used to develop age-invariant face recognition models. A key part of the research using individual volunteer subject images is highlighting the number of participating subjects, the number of face images originally acquired, the number of augmented face images, the median age [6], the minimum and maximum age and the separation between acquired images of each subject. The data augmentation process done on face images helps researchers work on the intra-class and inter-class variations sought for age-invariant face recognition [7]. The inter-class and intra-class variations help in the modeling of appropriate datasets for the development of age-invariant face recognition systems. The augmented face images are often used as data input to several deep learning models like the convolutional neural networks to create robust age-invariant face recognition systems [8].

In some applications, data augmentation is used to separate subject-specific facial features that are stable from variations in other facial features caused by aging [9]. This leads to the generation of ageinvariant face recognition systems that are robust to variations in facial features caused by aging. Data augmentation has been used to adapt face images for applications on mobile devices and cloud environments that operate in real-time [10]. The face images for such niche applications are, usually augmented to be compatible with mobile device applications. Data augmentation is done in various ways. Famous among them are rotation, the addition of noise, landmark perturbation and synthesis techniques [11]. Face images are augmented to dramatically increase their numbers, size and suitability for deep learning applications. Face images that are not augmented usually cause overfitting, pose variance, misalignment and illumination variations. The issue of illumination variations in face images is also addressed using data augmentation techniques like face lighting as seen in [12] to create robust face recognition systems. Sometimes data augmentation is done to avoid the need for paired face images and the true age of face image samples [13].

In this work improving the accuracy of an age invariant face recognition (AIFR) system using data augmentation technique on a classical pre-trained convolution neural network is the focus of this study. It AFIR system was achieved by augmenting the FG-Net dataset at the preprocessing phase with different types of noise that incidentally improved the accuracy of the system. This was largely because the pertained CNN adapted for training the proposed AIFR model was robust to noise.

\section{PROPOSED RESEARCH METHOD FOR THE IMPROVED AGING INVARIANT FACE RECOGNITION USING DATA AUGMENTATION}

This section describes the proposed research method for the improved age invariant face recognition using data augmentation. This network was designed to improve the recognition of the intra-class subject (same person) at different ages using data augmentation. The general procedure comprised of the same traditional steps: image acquisition, pre-processing, feature extraction, classification and system evaluation. In this work, the image pre-processing steps taken using data augmentation technique improved the performance of the system greatly. Four basic pre-processing steps were utilized. Feature extraction is the process of capturing the preferred trait descriptors but using the $\mathrm{CNN}$ instead of a handcrafted method. In this model, a pre-trained CNN architecture (Inception-ResNetv-2) was adopted. Classification is necessary 
to recognize the identity of the subject. This model utilized the Softmax classifier, which was used for multiclass classification. The system (model) performance was evaluated for its testing accuracy, testing loss, mean squared error and mean absolute error. The general processes for improved AIFR using data augmentation are shown in Figure 1 to Figure 3.

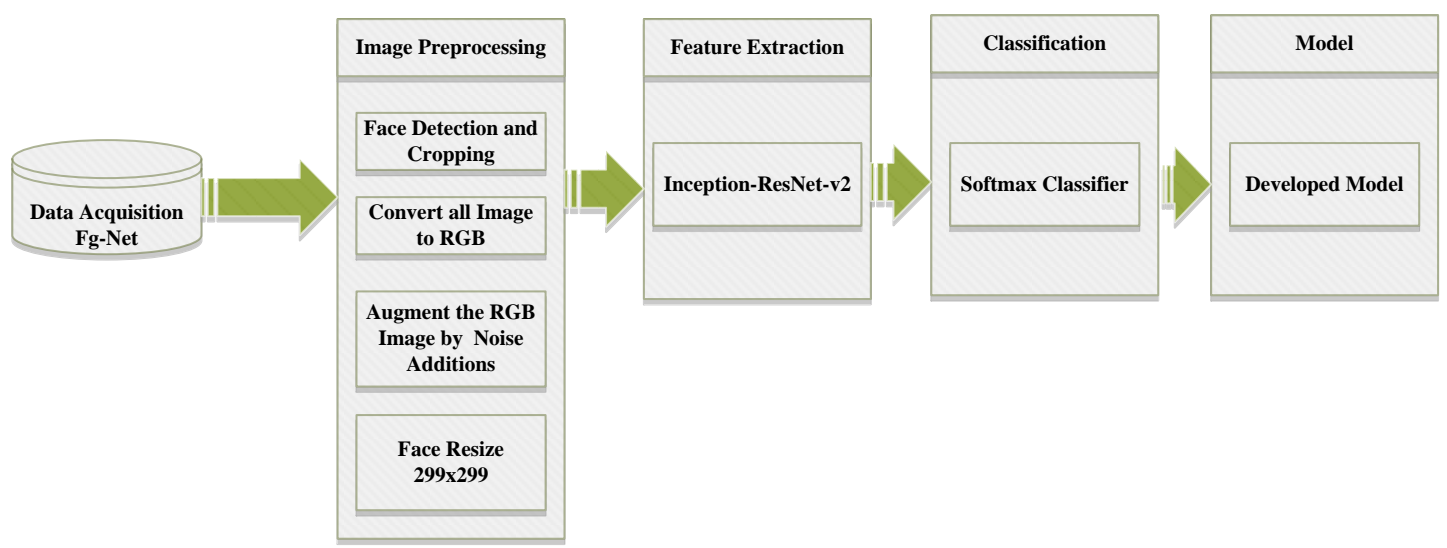

Figure 1. Basic block diagram for proposed age invariant face recognition training phase

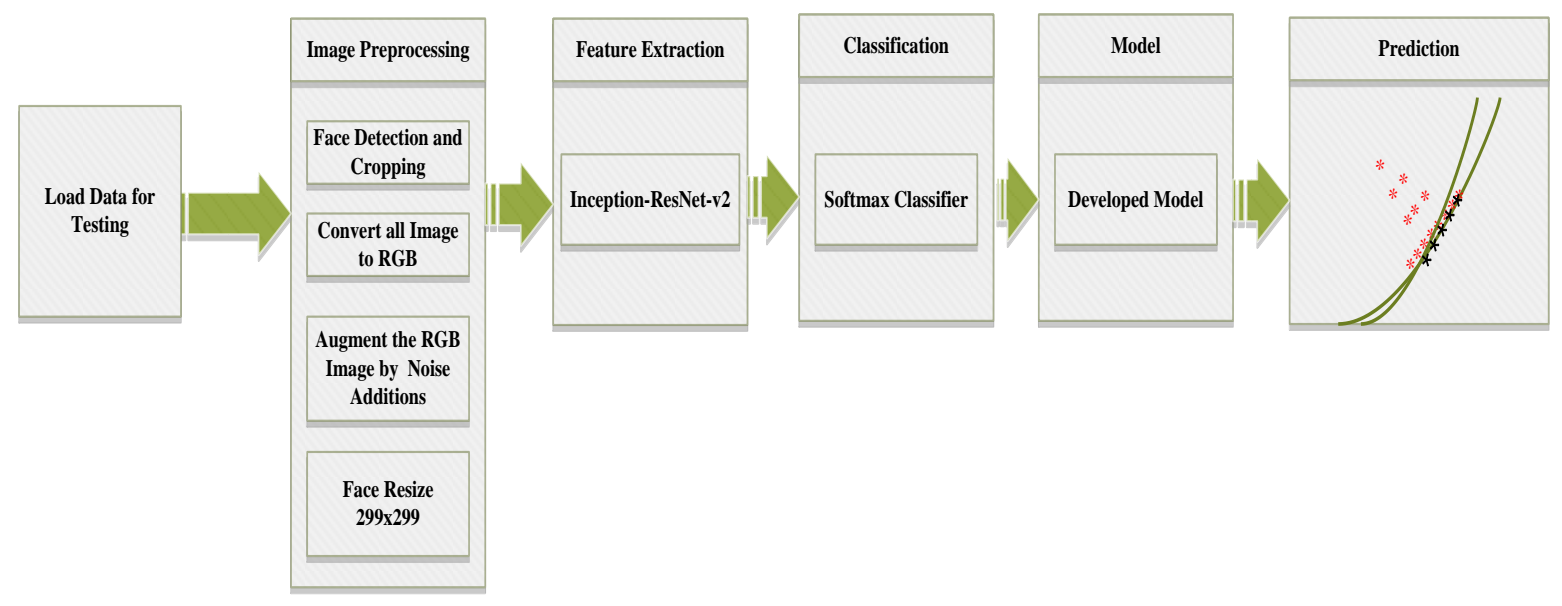

Figure 2. Basic block diagram for proposed age invariant face recognition testing phase

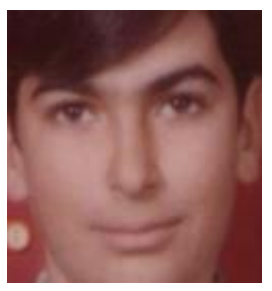

(a)

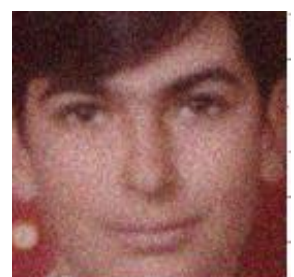

(b)

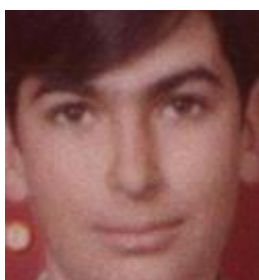

(c)

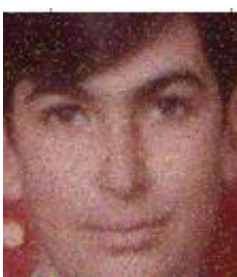

(d)

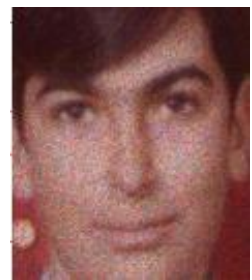

(e)

Figure 3. A sample cropped image from FG-net dataset with various noise addition: (a) Original image, (b) Image with Gaussian distribution noise, (c) Image with Poisson distribution noise, (d) Image with the distribution of salt \&pepper noise, (e) Image with the speckle noise distribution

\subsection{Data acquisition}

The face and gesture recognition network ageing database (FG-NET AD) contains 1002 images from 82 different subjects with age ranging from newborns to 69 years. However, ages up to 40 years are the most populated in the database. With the exception of recent images that were acquired digitally, other 
images in the database are the scanned photographs of subjects found in personal collections. Consequently, the quality of images in the database depends on the skill of the photographer, the imaging equipment used, the photographic paper used and the overall photograph condition. The images exhibit considerable variability in resolution, image sharpness, illumination, background, viewpoint and facial expression, which makes it highly challenging data for age-invariant face recognition. Furthermore, occlusions in the form of spectacles, facial hair and hats are also present in a number of images [14-19]. The FG-NET database also contains an average of between 10-15 face images of each subject at different ages [15]. The total number of subjects by gender are 34 males and 48 females. While the total number of images by gender is 395 males and 607 females. The database of the FG-NET AD was too small for deep learning experiments. Thus, the need for data augmentation.

\subsection{Training and testing the proposed AIFR model}

In this section, we shall consider; training the deep learning model using the transfer learning technique, using the trained deep learning model for adaptive face recognition in FG-NET and data augmentation.

\subsubsection{Training the deep learning model using transfer learning technique}

Transfer learning was used to train the Inception-ResNet-v2 network on the pre-processed FG-NET database for age-invariant face recognition. The training process was systematic and it is summarized thus:

a. Load the pre-processed FG-NET database into MATLAB using ImageDatastore object.

b. Split the database into a training set ( $80 \%$ images) and validation (testing) set (20\% images).

c. Resize all images in the train and test sets to $299 \times 299$ to make them compatible with the InceptionResNet-v2 network.

d. Load Inception-ResNet-v2 network in MATLAB.

e. Specify training options for transfer learning.

f. Re-train Inception-ResNet-v2 network on the train set of pre-processed FG-NET.

g. Test the trained network on the validation set.

h. Compute the network accuracy.

\subsubsection{Using the trained deep learning model for adaptive face recognition in FG-NET}

The trained deep learning model was used for testing the images from the FG-NET dataset by following the steps below:

a. Read an image from the FG-NET database in MATLAB.

b. Convert the image into an RGB format if it is a grayscale image.

c. Detect and crop the face of the subject in the image using the Viola-Jones Face Detector.

d. Resize the image to $299 \times 299$ to make it compatible with Inception-ResNet-v2.

e. Load the re-trained Inception-ResNet-v2 network from Section 2.2.1

f. Pass the image from step (d) to the re-trained network for class prediction.

g. Observe the prediction result and compare it to the ground truth.

\subsubsection{Data augmentation (the concept of image noise addition)}

Gaussian, Poisson, Salt \& Pepper, and Speckle noises were chosen to be added to the images from the FG-Net dataset [20]. Some amounts of these noises were added in each of the images in order to artificially inflate the datasets using label preservation transformation. Image noise addition and label preservation transformation are data augmentation techniques [21-30].

a. Image Gaussian noise addition

Gaussian noise modeling involves the addition of different random RGB values to each pixel in the image. The random values are mined from an arbitrary variable with mean $(\mu)$ value of zero and a variance 1.4 from normal density function. The mean and variance are selected to introduce a reasonable amount of noise. The mathematical model is as shown in (1) [31-33].

$$
\mathrm{P}(Z)=\frac{1}{\sqrt{2 \pi \delta}} e^{-(z-\mu) / 2 \delta^{2}}
$$

where $\mathrm{P}(Z)=$ Gaussian distribution noise in image, $\mu=$ Mean, $\delta=$ Standard deviation.

b. Image Poisson noise addition

Poisson noise is modeled by a Poisson procedure. Poisson noise is generated when a random variable is created for each pixel. The random variable has a Poisson distribution as shown in (2) [31-35]. An arbitrary sample is mined from every arbitrary variable. 


$$
\mathrm{P}(k)=\frac{e^{-\lambda}(\lambda)^{k}}{k !}
$$

where, $\mathrm{P}=$ Probability distribution,

$K=$ The number of Photons measured by a given sensor element,

$\lambda=$ Mean; that is equivalent to the value of the pixel.

c. Salt and pepper noise addition

Salt and Pepper noise can originate from transmission errors while carrying out analog-digital conversions. The salt and pepper noise was modeled by altering the value of each pixel of the image with a probability of 0.02 . The pixel was altered either to black, $(0,0,0)$ or white, $(255,255,255)$ in RGB values, both cases with a probability of 0.01 and 0.15 respectively. The Salt and Pepper mathematical model is as shown in (3) [24, 31, 36-39].

$$
\mathrm{P}(\mathrm{s})= \begin{cases}\mathrm{P}_{a} & \text { for } s=a \\ \mathrm{P}_{b} & \text { for } s=b \\ 0 & \text { otherwise }\end{cases}
$$

Where, $\mathrm{P}_{a} \mathrm{P}_{b}=$ Probability Density Function (PDF) of a and $\mathrm{b}$,

$\mathrm{P}(\mathrm{s})=$ Distribution of salt \&pepper noise in image,

$a, b=$ array image size

d. Speckle noise image addition

Speckle noise is a rough multiplicative noise. Speckle noise is generated by multiplying each pixel of the image by an arbitrary value. Arbitrary values are mined from an arbitrary variable with a mean 0.9 and a variance of 0.1 by a normal density function. The Speckle noise mathematical model is as shown in (4) $[24,31-34,40]$.

$$
g(x, y)=f(x, y) * \mu(x, y)+\xi(x, y)
$$

where, $g(x, y)=$ observed image,

$f(x, y)=$ multiplicative component

$\xi(x, y)=$ additive component of the speckle noise.

\subsection{Network architecture for feature extraction and classification}

The architecture adapted for this experiment is the Inception-ResNet-v2, which is a convolutional neural network that is trained on more than a million images from the ImageNet database and is used in ImageNet large-scale visual recognition challenge. The network is 164 layers deep. It can classify images into 1000 object categories and has an image input size of $299 \times 299$. In this research work, a pre-trained Inception-ResNet-v2 network was used. The convolutional neural network has already learned to extract powerful and informative features from natural images. It was used as a starting point to learn representative features from the FG-NET database using transfer learning for age invariant face recognition. The architecture was robust to noises used in the data augmentation stage [20,31,33,41]. The architecture of

\begin{tabular}{|c|c|c|c|c|c|c|c|c|}
\hline 1 & \multicolumn{2}{|l|}{ Convolution } & & $3 \times 3 / 1$ & $147 \times 147 \times 32$ & (32) & & \\
\hline 1 & \multicolumn{2}{|l|}{ Max Pooling } & & $3 \times 3 / 2$ & $73 \times 73 \times 160$ & & & \\
\hline 1 & \multicolumn{2}{|l|}{ Convolution } & & $3 \times 3 / 2$ & $73 \times 73 \times 160$ & (96) & & \\
\hline 1 & \multicolumn{2}{|l|}{ Convolution } & & $3 \times 3 / 1$ & $71 \times 71 \times 192$ & $(64,96)$ & $(64,64,64,96)$ & \\
\hline 2 & \multicolumn{2}{|l|}{ Inception-A } & 3 & & $35 \times 35 \times 256$ & (32) & $(32,32 / 2)$ & $(32,48,64 / 2)$ \\
\hline 3 & \multicolumn{2}{|l|}{ Reduction-A } & 3 & & $17 \times 17 \times 256$ & (384) & $(256,256,384)$ & \\
\hline 4 & \multicolumn{2}{|l|}{ Inception-B } & 3 & & $17 X 17 \times 896$ & (192) & $(128,160,192)$ & \\
\hline 5 & \multicolumn{2}{|l|}{ Reduction-B } & \multicolumn{2}{|l|}{3} & \multicolumn{4}{|c|}{$8 \times 8 \times 1792(256,384 / 2)(256,288 / 2)(256,288,320 / 2)$} \\
\hline 6 & \multicolumn{2}{|l|}{ Inception-C } & \multicolumn{2}{|l|}{3} & $8 \times 8 \times 1792(192)$ & \multicolumn{3}{|c|}{$(192,224,256)$} \\
\hline
\end{tabular}
the convolutional neural network is described in Table 1.

Table 1. A detail architecture of the convolution neural network (modified Inception-ResNet-v2) used for 


\section{RESULTS AND DISCUSSION}

In this section, the evaluation methodology and results of the research are given and a comprehensive discussion is made the comprehensive discussion.

\subsection{Evaluation methodology}

The performance evaluation metrics adopted in this work include testing accuracy, mean absolute error (MAE), mean square error (MSE), and loss function. These metrics are the most widely used for classification evaluation in the biometric and forensic analysis [2, 42-48].

a. Mean squared error

The MSE is a measure of the quality of a predictor, it is always non-negative, and values closer to zero are better. Where, $n$ in this case, is the number of iterations, $Y \omega$ is the training loss and $Y \phi$ is the testing loss. Therefore, MSE is computed as shown in (5) [49].

$$
M S E=\frac{1}{n} \sum_{i=1}^{n}(Y \omega-Y \phi)^{2}
$$

The mean squared error is the mean $\left(\frac{1}{n} \sum_{i=1}^{n}\right)$ of the squares of the errors $(Y \omega-Y \phi)^{2}$

b. Mean absolute error

The MAE is a measure of the dissimilarity between two variables. In this case between $Y \omega$ which is the training loss and $Y \phi$ which is the testing loss. $n$ is the number of iterations. Therefore, MAE is computed as shown in (6) [50].

$$
\operatorname{MAE}=\frac{\sum_{i=1}^{n}|Y \phi-Y \omega|}{n}
$$

The MAE is an average of the absolute errors $|\mathrm{Y} \phi-\mathrm{Y} \omega|$.

c. Loss function

Categorical cross-entropy and center loss were used as the loss functions for the improvement of the research model design. A loss function tells how good a classifier. Categorical cross-entropy is a loss function used to calculate the dissimilarity between two likely distributions. This dissimilarity is calculated for each point in the training and testing database. The mathematical expression used to evaluate the probability of dissimilarity is as shown in (7) [51, 52].

$$
\mathcal{L}_{\text {cross }}(y, \hat{y})=-\sum_{t=1}^{N} \cdot \sum_{i=1}^{C} y_{i}^{t} \cdot \log \left(\hat{y}_{i}^{t}\right)
$$

For an instant, $(\mathrm{x}, \mathrm{y})$ can be defined as, where: $x=$ input value, $y$ is true value, $\hat{\mathrm{y}}$ is predicted value by the system, $N$ is sum of iteration and $C$ is sum of class labels.

Wen et al.pproposed a loss function called center loss in addition to using the categorical cross-entropy loss. The notion is to increase the discriminative power of the totally learned features by decreasing the intra-class variations. The center loss function is as shown in [8].

$$
\mathcal{L}_{\text {center }(y, \hat{\mathrm{y}})}=\frac{1}{2} \sum_{t=1}^{N} \cdot \sum_{i=1}^{C}\left(\hat{\mathrm{y}}_{i}^{t}-c_{y_{i}}^{t}\right)^{2}
$$

While $c_{y_{i}}$ is the $y_{i}$ th class center of the features, $N$ is the number of iterations. Wen et al. observed that equation 8 does not attain the anticipated result. Two adjustments were made by Wen et al. to resolve this issue. The first adjustment is to bring up to date the centers based on a mini-batch as a replacement for the entire dataset. The second adjustment led to the introduction of two new variables, $\alpha$, and the $\delta$-function. $\alpha$ is used to regulate the learning rates of the centers and the $\delta$-function is a Boolean that results in 1 if the situation is true and 0 if the situation is false. The (9) defines the updated function of the class center.

$$
\Delta c_{j}(y, \hat{\mathrm{y}})=\frac{\sum_{t=1}^{N} \delta\left(y_{i}=j\right) \cdot\left(c_{j}-\hat{\mathrm{y}}_{i}\right)}{1+\sum_{t=1}^{N} \delta\left(y_{i}=j\right)}
$$


The new center of each class is as shown in (10)

$$
c_{j}^{t+1}=c_{j}^{t}-\alpha \cdot \Delta c_{j}^{t}
$$

While $\alpha \in[0,1]$, Wen et al., introduced $\lambda$ to balance the two-loss functions of the total loss function. The complete function is shown in (11).

$$
\mathcal{L}=\mathcal{L}_{\text {cross }}+\lambda \mathcal{L}_{\text {center }}
$$

In the event $\lambda$ is set to 0 , the total loss function is equal to the categorical cross-entropy function.

d. Accuracy

Where true positive (TP) symbolizes all experimented activities be appropriate to positive groups classified properly as positive groups. True negative (TN) are all experimented activities be appropriate to negative groups classified into negative groups. False-positive (FP) are all experimented activities be appropriate to negative groups being classified as positive groups, and false negative (FN) are all experimented activities be appropriate to positive groups being classified as negative groups.

$$
\text { Accuracy }=\frac{\mathrm{TP}+\mathrm{TN}}{\mathrm{TP}+\mathrm{TN}+\mathrm{FP}+\mathrm{TN}} * 100 \%
$$

e. System specification

The training and testing of the proposed model was successfully completed on a CPU with Core i7 processor and 64GB RAM in 10 hours using Matlab version 2018b software tools.

\subsection{Results}

In this section, the research results are presented and explained. Table 2 shows the performance of the proposed age invariant face recognition model. The performance of the proposed AFIR system was measured using accuracy, loss, mean squared error and mean absolute error. The training/testing accuracy and loss plot of the proposed AFIR model are shown in Figure 4 and Figure 5 respectively. Furthermore, the progression plot of square errors vs. iterations and absolute errors vs. iterations of the proposed AFIR model are shown in Figure 6 and Figure 7 respectively. The MSE and MAE is as calculated by (5) and (6).

Table 2. The performance of the proposed AIFR model

\begin{tabular}{cc}
\hline Parameters & Results \\
\hline Training accuracy & $100 \%$ \\
Testing accuracy & $99.94 \%$ \\
Training loss & $0.008 \%$ \\
Testing loss & $0.003 \%$ \\
Mean squared error (MSE) & 0.0158 \\
Mean absolute error (MAE) & 0.0637 \\
\hline
\end{tabular}

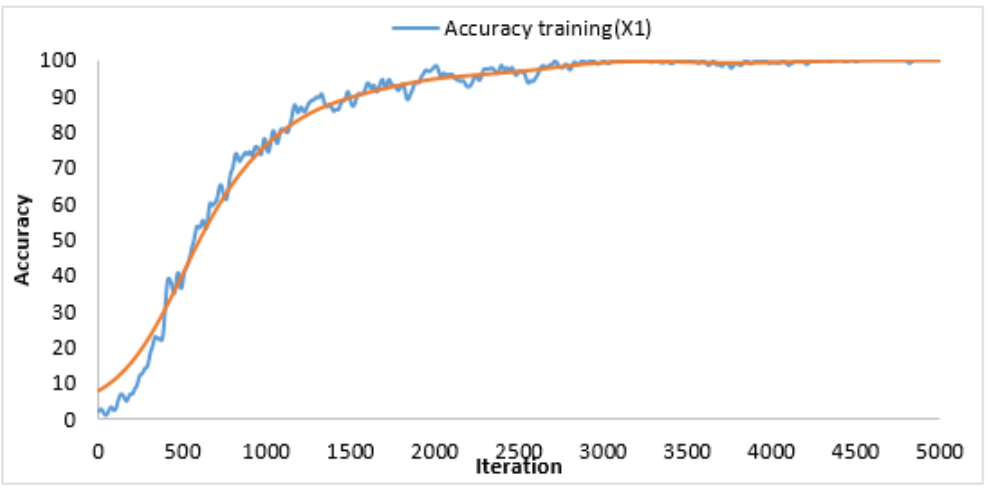

Figure 4. Training and testing accuracy plots of the proposed AFIR model 


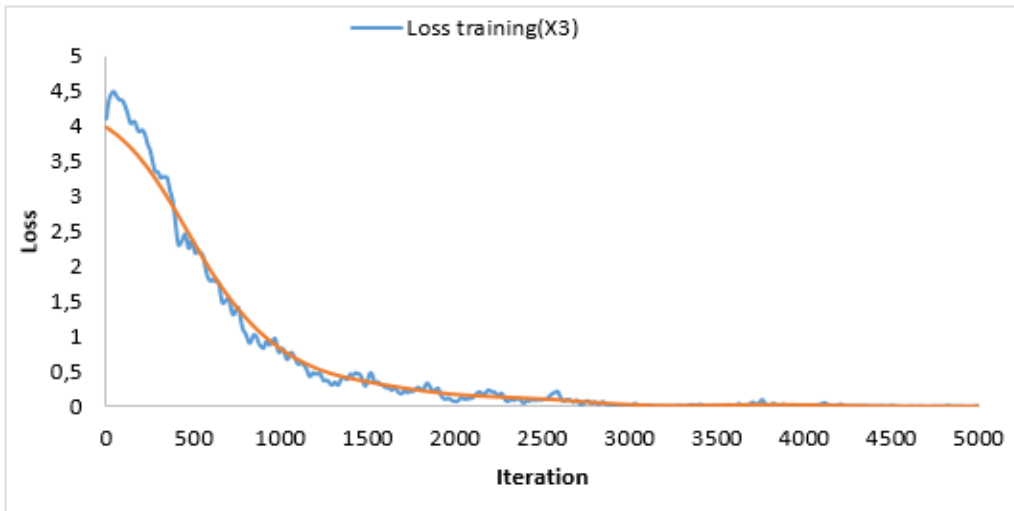

Figure 5. Training and testing loss (error function) plots of the proposed AFIR model

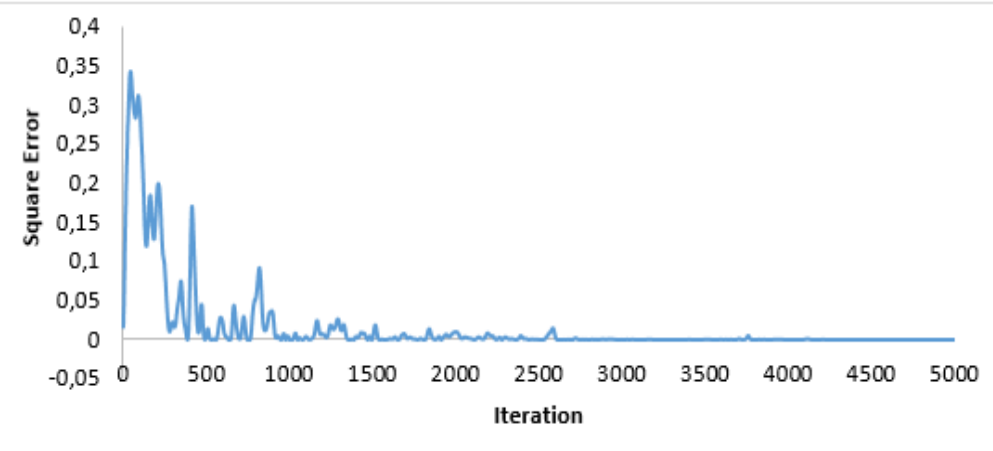

Figure 6. Progression plot of square errors vs. iterations of the proposed AFIR model

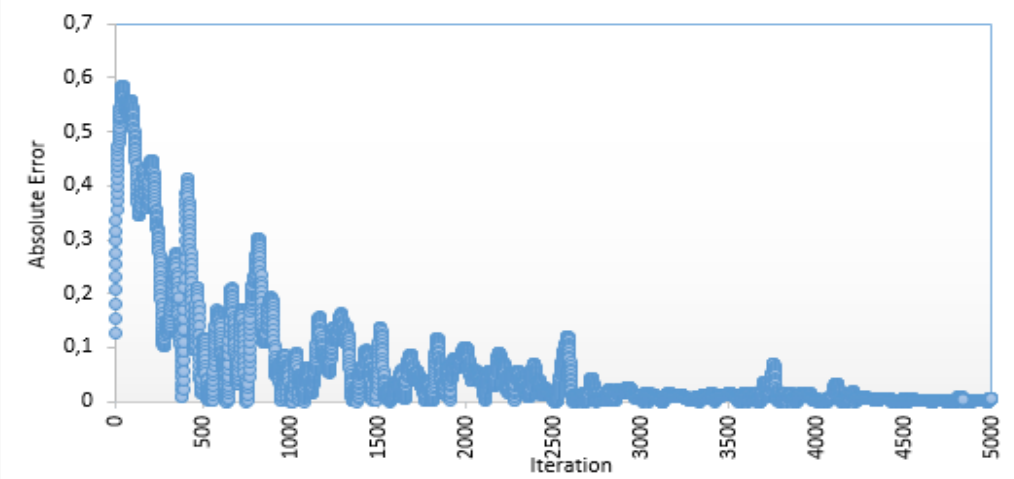

Figure 7. Progression plot of Absolute errors vs. iterations of the proposed AFIR mode

Table 2. The performance comparison of AIFR using mean absolute error (MAE)

\begin{tabular}{|c|c|c|c|c|c|}
\hline No. & Publication & Face dataset/dataset size & Algorithm & Evaluation protocol & $\begin{array}{c}\text { Performance } \\
\text { evaluation (MAE) }\end{array}$ \\
\hline 1 & Proposed & $\begin{array}{c}\text { FG-NET/1002 } \\
\text { (using-data argumentation technique) }\end{array}$ & $\begin{array}{l}\text { Classification } \\
\text { (CNN) }\end{array}$ & $20 \%$ test, $80 \%$ train data & MAE 0.0634 \\
\hline 2 & {$[53]$} & FG-NET/1002 & $\begin{array}{l}\text { Classification } \\
\quad(\mathrm{CNN})\end{array}$ & N/A & MAE 2.8 \\
\hline 3 & [54] & FG-NET/1002 & Classification & Leave-one-person-out & MAE 3.31 \\
\hline 4 & [55] & FG-NET/1002 & Classification & $98.8 \%$ train, $1.2 \%$ test & MAE 4.5 \\
\hline 5 & [56] & FG-NET/1002 & Classification & Leave-one-person-out & MAE 4.8 \\
\hline 6 & {$[57]$} & FG-NET/1002 & Classification & $20 \%$ test, $80 \%$ train & MAE 4.5 \\
\hline
\end{tabular}


The performance comparison of Age Invariant Face Recognition (AIFR) system using percentage accuracy is as shown in Table 3.

Table 3. The performance comparison of AIFR using percentage accuracy

\begin{tabular}{|c|c|c|}
\hline Author & Study/methodology & Results on percentage accuracy \\
\hline Proposed & $\begin{array}{l}\text { An Improved Age Invariant Face Recognition Using Data } \\
\text { Augmentation }\end{array}$ & $\begin{array}{l}\text { The proposed model achieved a recognition } \\
\text { accuracy of } 99.94 \% \text {. }\end{array}$ \\
\hline$[58]$ & $\begin{array}{l}\text { Age-invariant face recognition system based on identity } \\
\text { inference from appearance age }\end{array}$ & $\begin{array}{l}\text { The investigational study of face recognition was } \\
\text { done using FGNET data. Experimental results } \\
\text { achieved on AG-IIM showed training and } \\
\text { verification accuracies of } 89.8 \% \text { and } 88.2 \% \\
\text { respectively. }\end{array}$ \\
\hline [59] & $\begin{array}{l}\text { Age-invariant face recognition system using combined shape } \\
\text { and texture features. This technique merged texture and shape } \\
\text { feature sets to achieve age invariant facial recognition. }\end{array}$ & $\begin{array}{l}\text { The method realized an overall verification } \\
\text { accuracy of } 93 \% \text { on FG-NET AD. }\end{array}$ \\
\hline$[60]$ & $\begin{array}{l}\text { Feature-aging for age-invariant face recognition". This } \\
\text { method was used to forecast the aging of face structure in } \\
\text { order to improve the consequence of age advancement on } \\
\text { face recognition. }\end{array}$ & $\begin{array}{l}\text { Face recognition rates }(\%) \text { of the Gabor methods } \\
\text { and Age-invariant methods with face images from } \\
\text { different age groups show the best performance of } \\
32.7 \% \text { on testing accuracy. }\end{array}$ \\
\hline$[61]$ & $\begin{array}{l}\text { Age invariant face recognition and retrieval system using } \\
\text { coupled auto-encoder networks (CAN) }\end{array}$ & $\begin{array}{l}\text { Recognition rates of this method equated with state- } \\
\text { of-the-art algorithms on FGNET gave } 86.5 \% \\
\text { recognition rate }\end{array}$ \\
\hline$[62]$ & A geometrical approach for age-invariant face recognition & $\begin{array}{l}\text { The scheme was capable of attaining a determined } \\
\text { classification accuracy of } 99 \% \text { on FGNET database }\end{array}$ \\
\hline$[63]$ & $\begin{array}{l}\text { Face recognition across time-lapse using convolutional neural } \\
\text { networks }\end{array}$ & $\begin{array}{l}\text { Time-lapse run on FG-NET using numerous } \\
\text { cataloging techniques like nearest neighbor, linear } \\
\text { discriminant and subspace discriminant obtained } \\
\text { results of } 70.4 \%, 78.4 \% \text {, and } 80.6 \% \text { respectively }\end{array}$ \\
\hline$[64]$ & $\begin{array}{l}\text { Age invariant face recognition based on texture embedded } \\
\text { discriminative graph model. This proposed model takes full } \\
\text { advantage of the information of texture variations and } \\
\text { geometry topology contained in face images. }\end{array}$ & $\begin{array}{l}\text { Experimentations were done on the FG-NET aging } \\
\text { database, and a recognition accuracy of } 64.47 \% \text { was } \\
\text { achieved }\end{array}$ \\
\hline
\end{tabular}

\subsection{Discussion}

From the results presented in subsection 3.2, it is observed that the testing accuracy of the proposed model is $99.94 \%$. This signifies that if this model is embedded into a smart surveillance camera, it could correctly identify the face of the same subject across large age variation (in this experimental setup an average of 25 years was used) with $99.94 \%$ accuracy. Other metrics used in the measurement of the model are testing error of $0.04 \%$. The mean squared error is 0.0153 , while the mean absolute error is 0.0637 with a maximum iteration setting of 5000 and epochs of 50. Details of these metrics interpretation and relevant areas presented in subsection 3.1. The proposed AIFR model performance metrics outperform the results recorded in the literature to the best of our knowledge when compared to others using a similar dataset.

One of the novelties of this research work is that different types of noise augmentation were used to improve the accuracy of the AIFR system, as against the traditional practice of noise elimination at the preprocessing phase in order to improve the output accuracy. It is observed from these results that this new technique is more efficient form AIFR systems. The results show that a generic pre-trained classical CNN architecture (Inception-ResNet-v2) can be adapted in the AIFR domain. Thus saving processing time, computing resources and acquisition of huge training data that are not readily available in this AIFR domain. From the result obtained it is observed that the experimental design at the pre-processing phase greatly impacts the quality of the feature extracted and output of the classifier. Thus in this paper, the final result of the AIFR system is greatly improved as compared to other related works.

\section{CONCLUSION}

In this paper, a novel methodology of improving the accuracy of age invariant face recognition using noise augmentation technique and adapting a pre-trained deep convolution neural network (DCNN) was proposed. Experimentation was performed on the FG-Net dataset. The FG-Net dataset was augmented at the preprocessing stage using four types of noises to improve the features extracted and get a better classification. The augmented data was used to build an age invariant face recognition model. The model on testing was found to be very accurate in comparison to similar research works carried out on the same dataset. 


\section{ACKNOWLEDGEMENTS}

We acknowledged the sponsorship of the Covenant University Centre for Research Innovation and Discovery (CUCRID), Ota, Ogun State, Nigeria.

\section{REFERENCES}

[1] M. Sajid et al., "Data Augmentation-Assisted Makeup-Invariant Face Recognition," Mathematical Problems in Engineering, vol. 2018, 2018.

[2] Y. Li, G. Wang, L. Nie, Q. Wang, and W. Tan, "Distance metric optimization driven convolutional neural network for age invariant face recognition," Pattern Recognit., vol. 75, pp. 51-62, 2018.

[3] K. Okokpujie, S. John, C. Ndujiuba, and E. Noma-Osaghae, "Development of an Adaptive Trait-Aging Invariant Face Recognition System Using Convolutional Neural Networks," Information Science and Applications, vol 621, pp. 411-420, 2020.

[4] A. Yousaf, M. J. Khan, M. J. Khan, A. M. Siddiqui, and K. Khurshid, "A robust and efficient convolutional deep learning framework for age-invariant face recognition,” Expert Syst., vol. 37, no. 3 p. 12503, 2017.

[5] K. Okokpujie and S. Apeh, "Predictive Modeling of Trait-Aging Invariant Face Recognition System Using Machine Learning," Information Science and Applications, pp. 431-440, 2019.

[6] J. Deng, Y. Zhou and S. Zafeiriou, "Marginal Loss for Deep Face Recognition," 2017 IEEE Conference on Computer Vision and Pattern Recognition Workshops (CVPRW), Honolulu, HI, pp. 60-68, 2017.

[7] Y. Wen, Z. Li and Y. Qiao, "Latent Factor Guided Convolutional Neural Networks for Age-Invariant Face Recognition," 2016 IEEE Conference on Computer Vision and Pattern Recognition (CVPR), Las Vegas, NV, pp. 4893-4901, 2016.

[8] T. Zheng, W. Deng and J. Hu, "Age Estimation Guided Convolutional Neural Network for Age-Invariant Face Recognition," 2017 IEEE Conference on Computer Vision and Pattern Recognition Workshops (CVPRW), Honolulu, HI, pp. 503-511, 2017.

[9] P. Wang, W. Lin, B. Wu, K. Chao and C. Lo, "A Cross-Age Face Recognition Approach Using Fog Computing Architecture for User Authentication on Mobile Devices," 2018 IEEE 15th International Conference on e-Business Engineering (ICEBE), Xi'an, pp. 86-93, 2018.

[10] J.-J. Lv, X.-H. Shao, J.-S. Huang, X.-D. Zhou, and X. Zhou, "Data augmentation for face recognition," Neurocomputing, vol. 230, pp. 184-196, 2017.

[11] H. A. Le and I. A. Kakadiaris, "Illumination-Invariant Face Recognition With Deep Relit Face Images," 2019 IEEE Winter Conference on Applications of Computer Vision (WACV), Waikoloa Village, HI, USA, pp. 2146-2155, 2019.

[12] J. Zhao et al., "Look Across Elapse: Disentangled Representation Learning and Photorealistic Cross-Age Face Synthesis for Age-Invariant Face Recognition," Proceedings of the AAAI Conference on Artificial Intelligence, vol. 33, pp. 9251-9258, 2019.

[13] G. G. Chrysos, Y. Panagakis, and S. Zafeiriou, "Visual Data Augmentation through Learning," arXiv Prepr. arXiv1801.06665, 2018.

[14] F. Juefei-Xu, Khoa Luu, M. Savvides, T. D. Bui and C. Y. Suen, "Investigating age invariant face recognition based on periocular biometrics," 2011 International Joint Conference on Biometrics (IJCB), Washington, DC, pp. 1-7, 2011.

[15] G. Panis, A. Lanitis, N. Tsapatsoulis and T. F. Cootes, "Overview of research on facial ageing using the FG-NET ageing database," in IET Biometrics, vol. 5, no. 2, pp. 37-46, 2016.

[16] K. Okokpujie et al., "An Enhanced Voters Registration and Authentication Application Using Iris Recognition Technology," Int. J. Civ. Eng. Technol., vol. 10, no. 02, pp. 57-68, 2019.

[17] K. Okokpujie, E. Noma-Osaghae, S. John and R. Oputa, "Development of a facial recognition system with email identification message relay mechanism," 2017 International Conference on Computing Networking and Informatics (ICCNI), Lagos, pp. 1-6, 2017.

[18] K. Okokpujie, E. Noma-Osaghae, S. John, K. Grace and I. Okokpujie, "A face recognition attendance system with GSM notification," 2017 IEEE 3rd International Conference on Electro-Technology for National Development (NIGERCON), Owerri, pp. 239-244, 2017.

[19] M. Nimbarte and K. Bhoyar, "Age invariant face recognition using convolutional neural network," International Journal of Electrical and Computer Engineering (IJECE), vol. 8, no. 4, pp. 2126-2138, 2018.

[20] B. Fu, X. Zhao, C. Song, X. Li, and X. Wang, "A salt and pepper noise image denoising method based on the generative classification," Multimed. Tools Appl., vol. 78, no. 9, pp. 12043-12053, 2019.

[21] X. Zheng, "Data Augmentation with Artistic Style," PhD Dissertation, University of Dublin, 2018.

[22] N. McLaughlin, J. M. Del Rincon and P. Miller, "Data-augmentation for reducing dataset bias in person reidentification," 2015 12th IEEE International Conference on Advanced Video and Signal Based Surveillance (AVSS), Karlsruhe, pp. 1-6, 2015,

[23] D. Mwiti, "Research Guide: Data Augmentation for Deep Learning [ Nearly ] Everything you need to know in 2019," [Online]. Available at: https://heartbeat.fritz.ai/research-guide-data-augmentation-for-deep-learning7f141fcc191c, pp. 1-15. 2019. Accessed 23 January, 2020.

[24] D. M. Montserrat, Q. Lin, J. Allebach and E. J. Delp, "Training Object Detection And Recognition CNN Models Using Data Augmentation,” Soc. Imaging Sci. Technol., pp. 27-36, 2017.

[25] L. Taylor and G. Nitschke, "Improving Deep Learning with Generic Data Augmentation," 2018 IEEE Symposium Series on Computational Intelligence (SSCI), Bangalore, India, pp. 1542-1547, 2018.

[26] Y. Song and Z. Lin, "Species recognition technology based on migration learning and data augmentation," 2018 th 
International Conference on Systems and Informatics (ICSAI), Nanjing, pp. 1016-1021, 2018.

[27] C. Shorten and T. M. Khoshgoftaar, "A survey on Image Data Augmentation for Deep Learning," J. Big Data, vol. 6, no. 60, pp. 1-48, 2019.

[28] M. B. Lee, Y. H. Kim and K. R. Park, "Conditional Generative Adversarial Network- Based Data Augmentation for Enhancement of Iris Recognition Accuracy," in IEEE Access, vol. 7, pp. 122134-122152, 2019.

[29] B. Leng, K. Yu, and J. Qin, "Data augmentation for unbalanced face recognition training sets," Neurocomputing, vol. 235, pp. 10-14, 2017.

[30] A. Mikołajczyk and M. Grochowski, "Data augmentation for improving deep learning in image classification problem," 2018 International Interdisciplinary PhD Workshop (IIPhDW), Swinoujście, pp. 117-122, 2018.

[31] D. Wang, H. Lu, and M. H. Yang, "Kernel collaborative face recognition," Pattern Recognit., no. 48, pp. 3025-3037, 2015.

[32] A. M. A. Selami and A. Fadhil, " A Study of the Effects of Gaussian Noise on Image Features," Kirkuk Univ. J. / Sci. Stud., vol. 11, no. April 2016, pp. 152-169, 2016.

[33] S. Kaur, "Noise Types and Various Removal Techniques," nternational J. Adv. Res. Electron. cs Commun. Eng., vol. 4, no. 2, pp. 226-230, 2015.

[34] V. Rohit and J. Ali, "A Comparative Study of Various Types of Image Noise and Efficient Noise Removal Techniques,” Int. J. Adv. Res. Comput. Sci. Softw. Eng., vol. 3, no. 10, pp. 2277-128, 2013.

[35] MatLab, "Preprocess Data for Domain-Specific Deep Learning Applications," [Online]. Available at: https://www.mathworks.com/help/deeplearning/ug/preprocess-data-for-domain-specific-applications.html. Accessed 15 December, 2019.

[36] K. Mitra, A. Veeraraghavan and R. Chellappa, "Robust RVM regression using sparse outlier model," 2010 IEEE Computer Society Conference on Computer Vision and Pattern Recognition, San Francisco, CA, pp. 1887-1894, 2010.

[37] M. S. Shakeel and K. M. Lam, "Deep-feature encoding-based discriminative model for age-invariant face recognition," Pattern Recognit., vol. 93, pp. 442-457, 2019.

[38] "MatLab." [Online]. Available at: https://www.mathworks.com/help/deeplearning/ug/preprocess-data-for-domainspecific applications.htmland, accessed 5 Decemeber 2019.

[39] R. Sharma and M. S. Patterh, "A new pose invariant face recognition system using PCA and ANFIS," Optik (Stuttg)., vol. 126, no. 23, pp. 3483-3487, 2015.

[40] L. Nanni, S. Brahnam, and G. Maguolo, Data Augmentation for Building an Ensemble of Convolutional Neural Networks, Innovation in Medicine and Healthcare Systems, and Multimedia, vol. 145, pp. 61-69, 2019.

[41] M. Pkavya, "How does salt and pepper noise occurs in an image," [Online]. Available at: https://dsp.stackexchange.com/questions/28920/how-does-salt-and-pepper-noise-occurs-in-an-image. Accessed 10 December, 2019

[42] D. Wang, C. Otto, S. Member and A. K. Jain, "Face Search at Scale : 80 Million Gallery," MSU Tech. Rep. MSUCSE-, pp. 1-14, 2015.

[43] C. Chen and A. Ross, "Matching thermal to visible face images using hidden factor analysis in a cascaded subspace learning framework," Pattern Recognit. Lett., vol. 72, pp. 25-32, 2016.

[44] S. Banerjee, W. J. Scheirer, K. W. Bowyer, and P. J. Flynn, "Fast Face Image Synthesis with Minimal Training," arXiv:1811.01474, pp. 1-11, 2018

[45] B. F. Klare and A. K. Jain, "Heterogeneous Face Recognition Using Kernel Prototype Similarities," in IEEE Transactions on Pattern Analysis and Machine Intelligence, vol. 35, no. 6, pp. 1410-1422, 2013.

[46] F. B. Alvi and R. Pears, "A composite spatio-temporal modeling approach for age invariant face recognition," Expert Syst. Appl., vol. 72, pp. 383-394, 2017.

[47] K. Okokpujie, E. Noma-osaghae, O. Okesola, O. Omoruyi, Osemwegie Chinonso, S. John, and I. P. Okokpujie, "Fingerprint Biometric Authentication Based Point of Sale Terminal," in K. J. Kim and N. Baek (eds.), Information Science and Applications 2018, Lecture Notes in Electrical Engineering, vol. 514, pp. pp 229-237, 2019.

[48] K. Okokpujie et al., "Integration of Iris Biometrics in Automated Teller Machines for Enhanced User Authentication," in Kim K., Baek N. (eds) Information Science and Applications 2018. ICISA 2018. Lecture Notes in Electrical Engineering, pp. 219-228, 2019.

[49] Q. Zhu, P. Zhang, and X. Ye, "A New Loss Function for CNN Classifier Based on Pre-defined Evenly-Distributed Class Centroids," arXiv1904.06008[cs.CV] 2019, pp. 1-10, 2019.

[50] R. Angulu, J. R. Tapamo, and A. O. Adewumi, Age estimation via face images: a survey. EURASIP Journal on Image and Video Processing, vol. 42, pp. 1-35, 2018.

[51] Y. Toda and F. Okura, "How Convolutional Neural Networks Diagnose Plant Disease," Plant Phenomics, vol. 2019, pp. 1-14, 2019.

[52] Y. Wen, K. Zhang, Z. L. B, and Y. Qiao, "A Discriminative Feature Learning Approach," 2016 European conference on computer vision, Amsterdam, pp. 499-515, 2016.

[53] Z. Hu, Y. Wen, J. Wang, M. Wang, R. Hong and S. Yan, "Facial Age Estimation With Age Difference," in IEEE Transactions on Image Processing, vol. 26, no. 7, pp. 3087-3097, 2017.

[54] I. Huerta, C. Fernández, C. Segura, J. Hernando, and A. Prati, “A deep analysis on age estimation," Pattern Recognit. Lett. J., vol. 68, pp. 239-249, 2015.

[55] H. Han and A. K. Jain, "Age, Gender and Race Estimation from Unconstrained Face Images," MSU Tech. Rep. MSU-CSE-14-5 Age, pp. 1-9, 2014.

[56] X. Geng, C. Yin and Z. Zhou, "Facial Age Estimation by Learning from Label Distributions," in IEEE Transactions on Pattern Analysis and Machine Intelligence, vol. 35, no. 10, pp. 2401-2412, 2013.

[57] G. Panis, A. Lanitis, N. Tsapatsoulis and T. F. Cootes, "Overview of research on facial ageing using the FG-NET 
ageing database," in IET Biometrics, vol. 5, no. 2, pp. 37-46, 2016.

[58] H. Zhou and K. M. Lam, "Age-invariant face recognition based on identity inference from appearance age," Pattern Recognit., vol. 76, pp. 191-202, 2018.

[59] A. S. Osman Ali, V. Sagayan, A. M. Saeed, H. Ameen and A. Aziz, "Age-invariant face recognition system using combined shape and texture features," in IET Biometrics, vol. 4, no. 2, pp. 98-115, 2015.

[60] H. Zhou, K. Wong and K. Lam, "Feature-aging for age-invariant face recognition," 2015 Asia-Pacific Signal and Information Processing Association Annual Summit and Conference (APSIPA), Hong Kong, 2015, pp. 1161-1165.

[61] C. Xu, Q. Liu, and M. Ye, "Age invariant face recognition and retrieval by coupled auto-encoder networks," Neurocomputing, 2017, vol. 222, pp. 62-71, 2017.

[62] A. S. O. Ali, V. S. Al Asirvadam, A. S. Malik, and A. Aziz, "A geometrical approach for age-invariant face recognition," Lect. Notes Comput. Sci. (including Subser. Lect. Notes Artif. Intell. Lect. Notes Bioinformatics), vol. 8237 LNCS, pp. 81-96, 2013.

[63] H. El Khiyari and H. Wechsler, "Face Recognition across Time Lapse Using Convolutional Neural Networks," Journal Informasi Security, vol.7, no.3, pp. 141-151, 2016.

[64] Hongyu Yang, Di Huang and Yunhong Wang, "Age invariant face recognition based on texture embedded discriminative graph model," IEEE International Joint Conference on Biometrics, Clearwater, FL, pp. 1-8, 2014.

\section{BIOGRAPHIES OF AUTHORS}

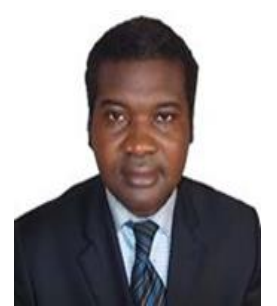

Kennedy Okokpujie holds a bachelor of engineering (B.Eng.) in electrical and electronics engineering, Master of Science (M.Sc.) in electrical and electronics engineering, Master of Engineering (M.Eng.) in electronics and telecommunication engineering and Master of Business Administration (MBA). He is currently lecturing in the Department of Electrical and Information Engineering at Covenant University, Ota, Ogun State, Nigeria. He is a member of the Nigeria Society of Engineers and the Institute of Electrical and Electronics Engineers (IEEE). His research areas of interest include biometrics, artificial intelligent, and digital signal processing. Email: kennedy.okokpujie@covenantuniversity.edu.ng

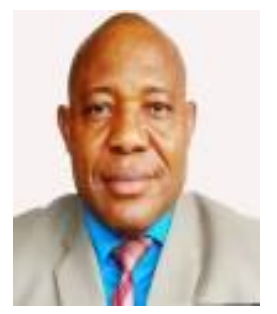

Samuel Ndueso John is a Professor of Computer Systems and Network Engineering. He has his higher education at Donetsk National Technical University, Ukraine where he successfully defended and obtained B.Sc, M.SC, MPhil and Ph.D. degrees in computer systems and network engineering, specializing in computer science, computing machines, complex systems, security and networks in 1993, 1994, 2000 and 2005, respectively. Presently, John is a Professor of Computer Systems and Network Engineering in the Department of Electrical/Electronic Engineering, Faculty of Engineering and Technology, Nigerian Defence Academy, Kaduna, Nigeria. John has acquired valuable knowledge and practical experience in the use of information technology as an enabler of industrial and national development goals. He has a vast knowledge of computing and has applied it in the pursuance of a wide range of indigenous ICT convergence, data efficiency management, cyber security, cybercrime and telemedicine solutions.

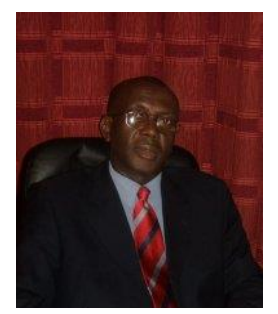

Charles U. Ndujiuba is a Professor of Communication Engineering with Air Force Institute of Technology Nigerian Air Force, Kaduna state, Nigeria. Prof. C. U. Ndujiuba holds a Ph.D. in electrical and electronics engineering from the University College London (University of London); Master Specialize (Masters with Specialization) in radio communications from Ecole Superieure d'Electricite (SUPELEC) France; MSc in electrical engineering from the University of Lagos, BSc in electronics and communications engineering from the London Metropolitan University. Prof. Ndujiuba is a Chartered Electronics Engineer (CEng) and a highly-skilled wireless professional. He has more than 25 years of RF, microwave, fixed-line (SDH and PDH), and PMR experience, with considerable international exposure. Prof. Ndujiuba has attended several conferences and published many technical papers in major professional journals. Prior to joining Covenant University Ota Nigeria in 2011, Ndujiuba was the Technical Director of Globe Trunk Ltd UK. His research interests include monolithic microwave integrated circuits (MMIC), active filters, ultra-low noise amplifiers, active devices and circuits, UWB Transmitter, modelling and simulation, dielectric resonator antennas, and detection and collision avoidance of unmanned aerial vehicles. 


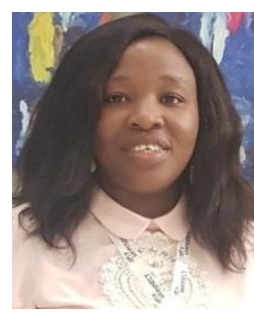

JOKE A. BADEJO received the B.Tech. degree from Ladoke Akintola University of Technology, Nigeria, in 2003, the M.Eng. and Ph.D. degree from Covenant University, Nigeria, in 2007 and 2015 respectively; all in Computer Engineering. She is currently an Assistant Professor in the Department of Electrical and Information Engineering at Covenant University, Nigeria. During her Ph.D., she received the International Association for Pattern Recognition and MORPHO grant in 2012 for Biometrics training. She was also a Visiting Doctoral Student at Biomedical Signal Analysis Laboratory at Clarkson University, Potsdam, NY, USA, in 2015. Her research interests include Biometrics \& Biomedical Image Analysis, Machine Learning, Data Analytics, Software Engineering. She has authored/co-authored about 35 scholarly articles in ISI/CPCI indexed journals and conference proceedings. She currently leads the Data Analytics team, which supports data-driven decision making in Covenant University. She is a member of several professional bodies such as the Council for the Regulation of Engineering in Nigeria (COREN) and the Institute of Electrical and Electronics Engineers (IEEE). Joke enjoys being an academic and loves contributing impactful and cost-effective solutions to current societal engineering problems in Africa.

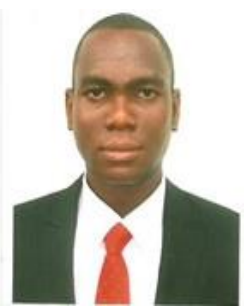

Etinosa Noma-Osaghae holds a master's degree in electronics and telecommunication engineering from the University of Benin. His areas of interest are telecommunication and signal processing. 